\title{
A Unique Type of Dural Arteriovenous Fistula at Confluence of Sinuses Treated with Endovascular Embolization: A Case Report
}

\author{
Rahul Gupta, MD1, 2, Shigeru Miyachi, MD1, Noriaki Matsubara, MD1, Takashi Izumi, MD', \\ Takehiro Naito, MD', Kenichi Haraguchi, MD', Toshihiko Wakabayashi, MD'1
}

Dural arteriovenous fistula (DAVF) is classically defined as abnormal arteriovenous connections located within the dural leaflets. Though the exact etiology is still not clear, they are generally accepted as acquired lesions. However, some DAVFs formed as the congenital disorders are called dural arteriovenous malformations and these lesions with a marked cortical venous reflux are considered to be aggressive and warrant an early intervention. The authors describe a case of 35-year-old man presented with unique type of DAVF. The fistula was located adjacent to the confluence of venous sinuses with multiple feeders. The feeders drained into a large venous pouch just anterior to the confluence which had a bilateral venous drainage. This was associated with multiple cerebellar venous ectasia along the draining cortical vein. It was managed by staged endovascular procedures and complete cure could be achieved. The pathogenesis and technique of embolization of this complex fistula/malformation are also discussed.

Key Words : Dural arteriovenous fistula; Confluence of sinuses; Embolization; Cortical reflux; Varix

Although early case reports appeared in the 1930s, the concept of spontaneous dural arteriovenous fistulas (DAVF) in adults was first introduced in 1951 and in children in 1964 [1]. DAVF is classically defined as abnormal arteriovenous connections between an

\footnotetext{
'Department of Neurosurgery, Nagoya University Graduate School of Medicine, Nagoya, Aichi, Japan

2Department of Neurosurgery, G B Pant Hospital, Maulana Azad Medical College, University of Delhi, New Delhi, India.

Received August 12, 2012; accepted after revision October 12, 2012.

Correspondence to: Shigeru Miyachi, MD. Associate Professor, Department of Neurosurgery, Nagoya University Graduate School of Medicine, 65 Tsurumai-cho, Showa-ku, Nagoya, Aichi 466-8550, Japan.

Tel. 81.52.744.2353 Fax. 81.52.744.2360

E-mail: smiyachi@med.nagoya-u.ac.jp

This is an Open Access article distributed under the terms of the Creative Commons Attribution Non-Commercial License (http://creativecommons.org/licenses/by-nc/3.0) which permits unrestricted non-commercial use, distribution, and reproduction in any medium, provided the original work is properly cited.
}

arterial feeder and a dural venous sinus or leptomeningeal vein with the channels located within the dural leaflets. They have many different anatomical features and are present at different locations in the brain. This makes the nomenclature a little confusing. A DAVF draining primarily into the transverse sinus has been labelled a dural transverse sinus fistula in the literature. However, the tentorial DAVF is another subset of intracranial DAVF where the fistula is in the tentorium [2]. The tentorial medial type has the fistula located adjacent to torcula or straight sinus and primarily drains into infratentorial veins apart from the venous sinus. Some DAVFs formed as the congenital disorders are called dural arteriovenous malformations and these lesions with a marked cortical venous reflux are considered to be aggressive and warrant an early intervention and obliteration. Here, we report a case of congenital DAVF treated by a combination of transarterial and transvenous endovascular embolization. The fistula was located just anterior to the confluence of 
sinuses and is having feeders from dural and tentorial arteriesand the fistula sac drains into the confluence and had a cerebellar cortical venous reflux.

\section{CASE REPORT}

\section{Preoperative Course and Examination}

A 35-year-old male, who suffered from tinnitus in the right side since childhood, presented in emergency with a history of sudden and severe headache of two hours duration. There was no associated relevant history and he was alert and oriented. There was slight nuchal rigidity and bruit could be heard in the occipital region on both sides. Rest of the clinical examination was normal.

Computed tomography scan done immediately showed diffuse subarachnoid haemorrhage (SAH) along the inferior surface of the tentorium. Magnetic resonance imaging (MRI) showed abnormal flow voids just anterior to the torcula and in the left cerebellar hemisphere. There were many dilated veins connecting these flow voids. There was no associated infarct or edema in the brain parenchyma (Fig. 1).

Intracranial angiogram revealed a DAVF adjacent to the confluence of venous sinuses and an associated large focal venous ectasia (varix) near the torcula (Fig. 2 ). It was supplied from carotid vessels and vertebral arteries. The main feeders were from both of occipital, ascending pharyngeal, middle meningeal and posterior meningeal arteries. These feeders converged into a big varix just anterior and to the right of the confluence and had two drainage outlets. The retrograde flow or reflux wandered into the left cerebellar cortical veins and multiple varices formation in the left cerebellar hemisphere whereas the other was an antegrade flow into the dominant right transverse sinus via a small channel.

\section{Intervention}

The patient was initially managed in another hospital by endovascular procedure for flow reduction, where the main feeders were palliatively embolized using particles via the transarterial route. Feeders from right occipital, right middle meningeal and left occipital arteries were embolized and the main residual feeder was the left ascending pharyngeal artery. The patient's symptom was improved and his tinnitus reduced in intensity. Since there was a significant residual lesion, the patient was referred to our hospital for complete obliteration of the fistula.

Transvenous embolization (TVE) was performed through the transfemoral approach six weeks after the first procedure. The procedure was performed under local anaesthesia and moderate sedation with Propofol (Diprivan, AstraZeneca K.K., Osaka, Japan). A coaxial system was used to cannulate the transverse sinus. A 6 Fr, $90 \mathrm{~cm}$ guiding sheath (Shuttle-SL; Cook, Bloomington, IN, USA) and $6 \mathrm{Fr}, 100 \mathrm{~cm}$, curved tip guiding catheter (Envoy MPC, Cordis, Miami, FL, USA) were placed in the internal jugular vein. Through this 6 Fr guiding catheter, a 4 Fr, $125 \mathrm{~cm}$ guiding catheter (Cerulean G, Medikit, Tokyo, Japan) was placed in the right transverse sinus (Fig. 3A). The Excelsior 1018 Microcatheter (Boston Scientific, Natick, MA, USA) was carefully placed at the origin of the left cerebellar cortical vein. The preferential and rapid flow into the refluxed vein helped us to easily direct the tip of the microcatheter. The distal end of this vein was occluded with 8 coils of various sizes from different companies. Complete obliteration of flow to
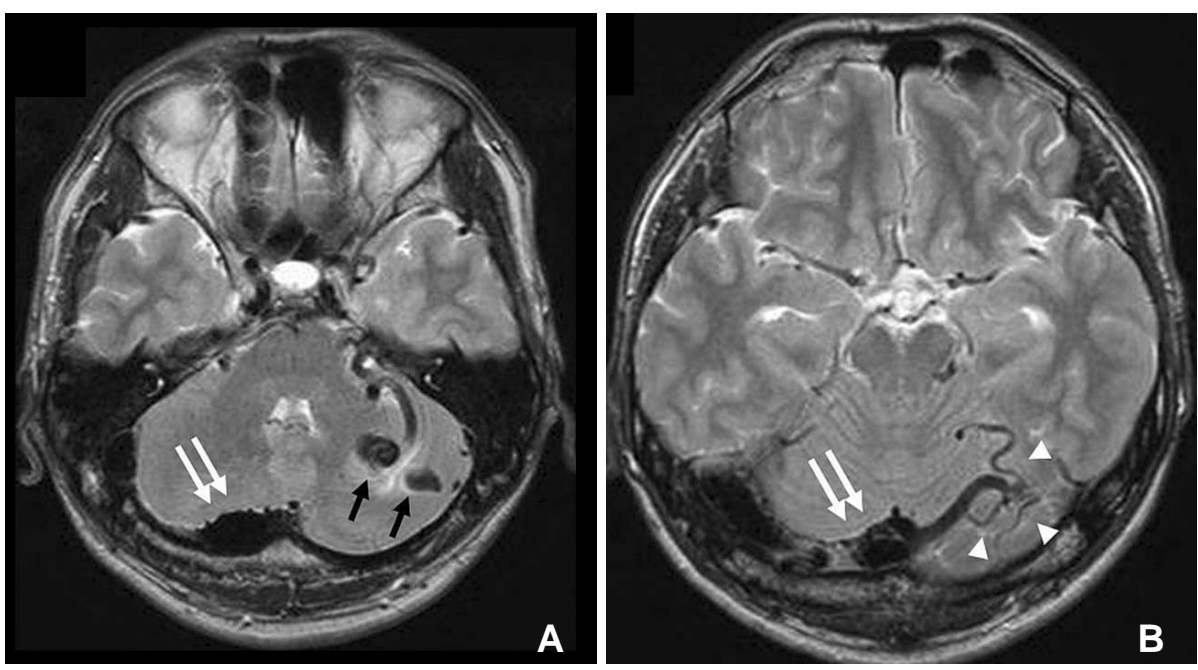

Fig. 1. T2 weighted MR images (A, B) showing large varix near the torcula (double arrows), multiple cerebellar varices (black arrows) and draining cortical cerebellar veins (arrow heads). 


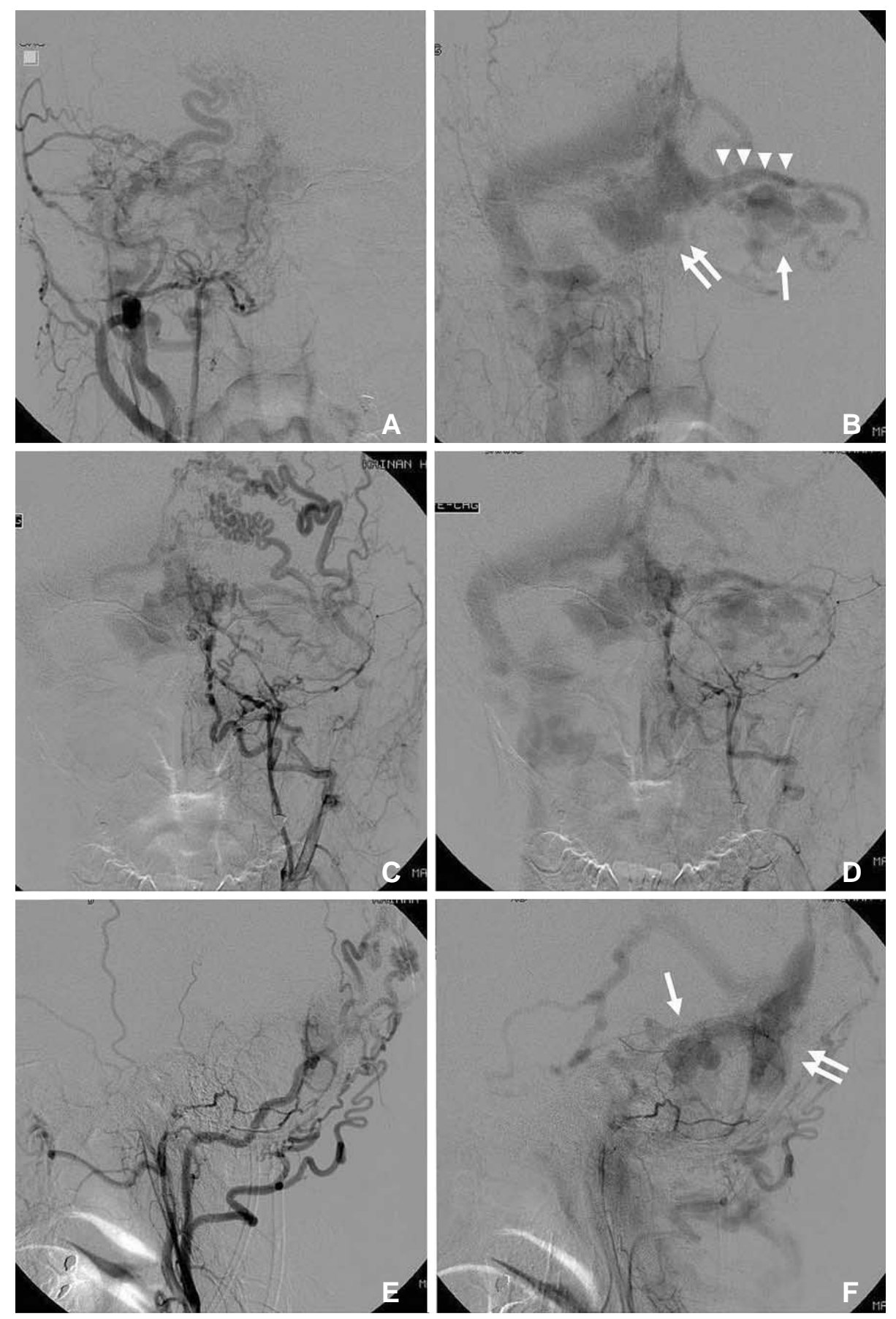

Fig. 2. Preoperative angiograms. A, B. Right external carotid angiogram of anterior-posterior (A-P) view: early phase (A) and late phase (B). C-F. Left external carotid angiogram: A-P view in early phase (C) and late phase (D). Lateral view in early phase (E) and late phase (F). Multiple feeders from both ECAs are demonstrated. Main feeders are bilateral ascending pharyngeal and occipital arteries. Right transverse sinus is dominant and enlarged. Large varix near the torcula (double arrows), cerebellar varices (arrow) and abnormaly dilated draining cortical cerebellar veins (arrow heads) are apparently shown on angiograms in late phase.

the cerebellar varices was obtained (Fig. 3B). Then the main varix was filled with 26 coils with an aim to block all the shunt points (Fig. 3C, D).

In spite of dense packing seen on imaging, there were residual shunt points which allowed the flow of arterial blood into the transverse sinus. Booster transarterial embolization with polyvinyl alcohol (PVA) particles and microfibrous collagen was then performed through the left external carotid artery (ECA). A complete occlusion of DAVF could be achieved (Fig. 3E, F).

\section{Postoperative Course}

The postoperative period was uneventful. The symptom of tinnitus was entirely disappeared. MRI acquired 5 days after procedure showed thrombosis of the all the varices and cerebellar cortical vein without any edema or infarction (Fig. 4).

\section{DISCUSSION}

DAVF in adulthood is usually considered to be an 
acquired and progressive disease [3] and there is no relationship to the hereditary or developmental disorders. But there are a few case reports of DAVF in neonates and childhood which indicate a congenital origin $[4,5]$. A long standing history of tinnitus in the present case might point towards the congenital origin of this DAVF. However, the lesion remained dormant for so many years without symptom. The formation of many cerebellar varices suggests a cortical venous reflux and asymptomatic chronic venous congestion. Thus, a delicate balance between the inflow of arterial blood and the venous outflow contained the disease.

The location of the varix was just anterior and to right side of the confluence of sinuses, probably in the subdural space. The wall of the varix abutted the cerebellar convexity dura as well as the tentorium. It received feeders from the meningeal branches of ECA and vertebral arteries and tentorial branches of the
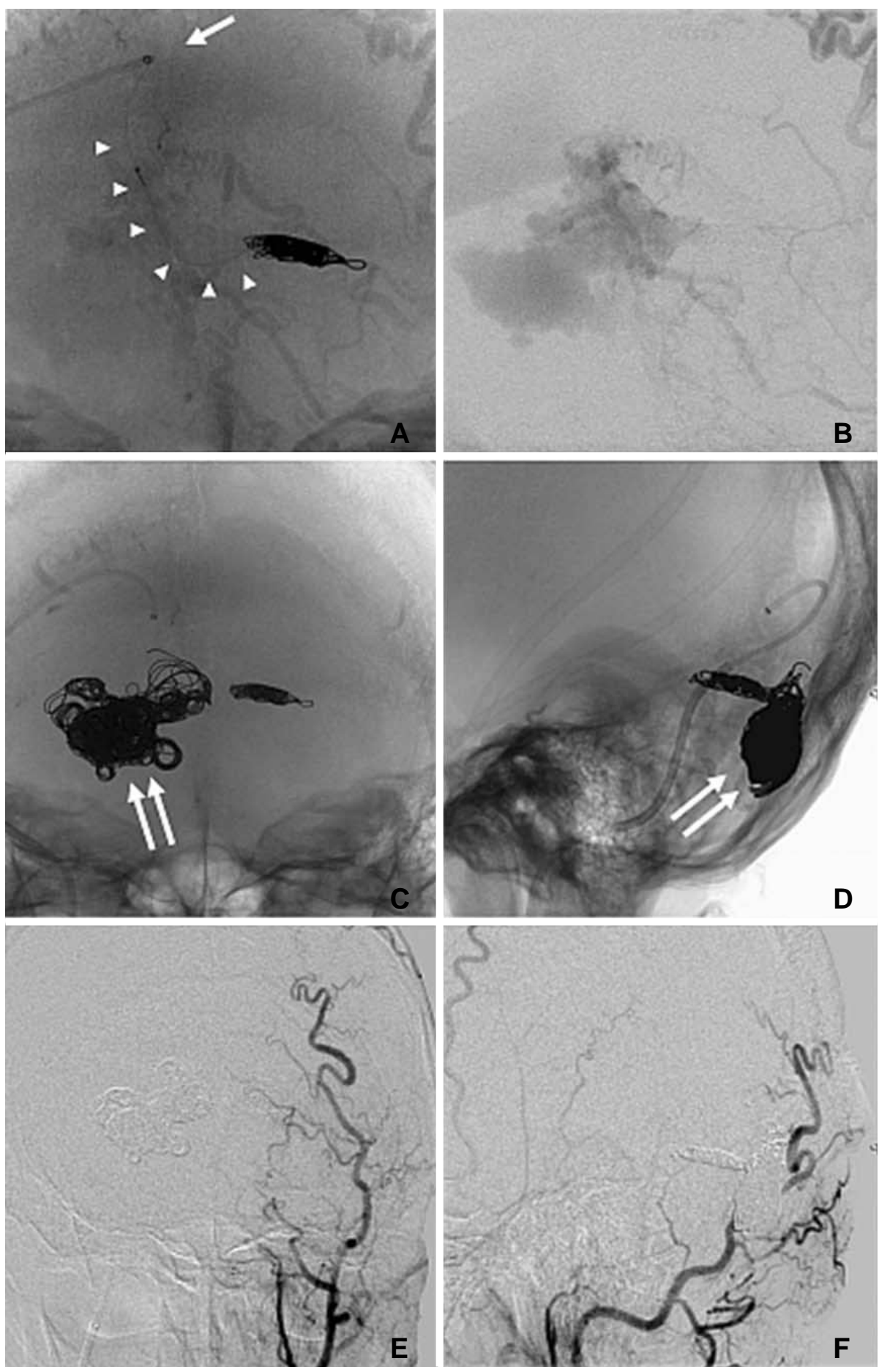

Fig. 3. A. Skull X-ray during procedure in anterior-posterior $(A-P)$ view showing coil mass placed in dilated cerebellar cortical vein. Tip of 4Fr guiding catheter in transverse sinus (arrow) and the course of microcatheter in the venous system (multiple arrows) are also displayed. B. Angiogram acquired after coil embolization of abnormal cerebellar vein. Cortical venous reflux of cerebellum disappeared although large varix near the torcula still remained. C, D.Skull X-ray A-P $(\mathbf{C})$ and lateral (D) views showing coil mass filling that varix (double arrows) and cortical vein. E, F. Left external carotid angiogram after embolization in A-P (E) and lateral views (F) showing complete occlusion of DAVF. 

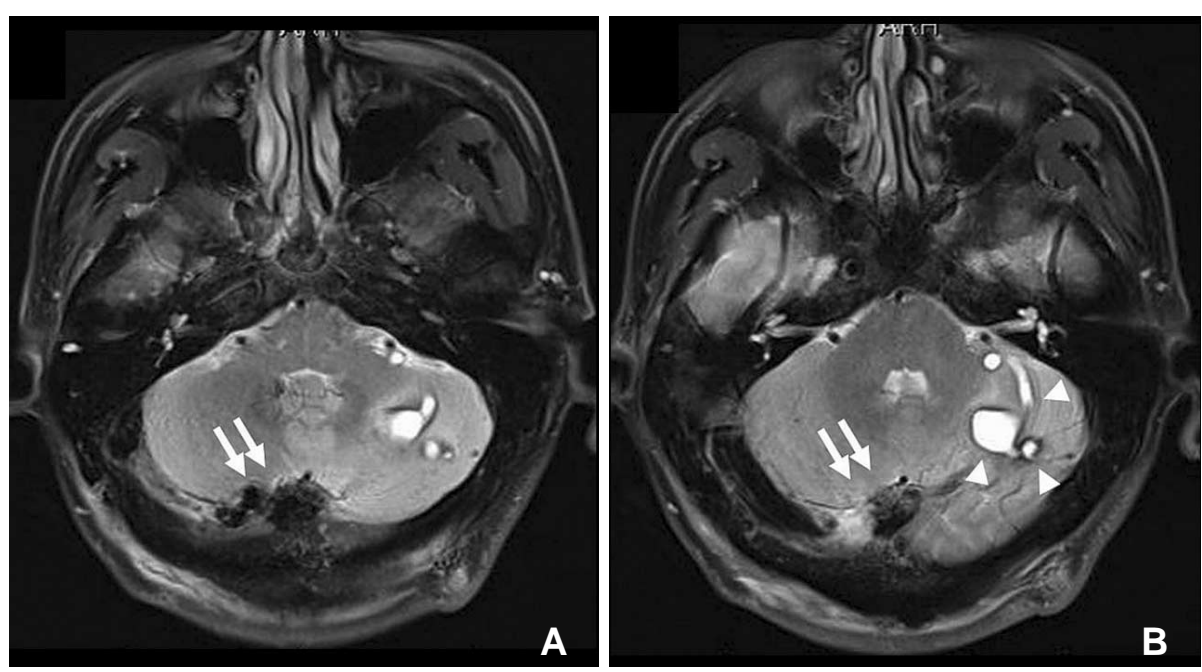

Fig. 4. Postprocedural T2 weighted MR images (A, B) showing metal artefacts in large varix (arrows) and thrombosis of cerebellar varices and draining vein (arrow heads).

internal carotid artery (ICA). Hence the fistulous points were located both in the convexity dura as well as the tentorium. It drained into the dominant right transverse sinus and left cortical vein. Since, it cannot be categorised as a tentorial DAVF or a dural transverse sinus fistula alone, we have labelled it as a DAVF of the confluence.

DAVF with leptomeningeal venous drainage or an associated varix are defined as high risk and are associated with a dismal natural history [3]. These high risk fistulas typically present with hemorrhage or progressive neurological deficits. Because the draining veins transverse the various compartments from the dura to the brain parenchyma, their rupture may create subdural, subarachnoid or intracerebral hemorrhage. In the present case, the cerebellar cortical vein which was the major drainage route must have burst in the subarachnoid space leading to subarachnoid bleeding. Parenchymal hematoma is more often reported with similar type of DAVF and the varices in the cerebellar hemisphere were a potential source like present case. But the unique flow dynamics made the cortical vein vulnerable to rupture. Similar arterialised arachnoid draining veins at a distance from the nidus have been attributed for hemorrhage [6]. In the literature, the angiographic features associated with the aggressive behaviour of the DAVFs include location at the tentorial incisura, leptomeningial or Galenic venous drainage and presence of venous aneurysms [6].

DAVF in the present case had a unique arterial feeder and venous drainage pattern which developed over the years. There were multiple feeders in dura and tentorium from all the major cranial arteries which opened into a large bowl like varix located in the subdural space. The varix was actually the pouch like dilated terminal portion of the cortical vein which had grown in size over the years due direct feeders. It had a distal and proximal connection to the normal vein. The normal antegrade drainage via a small channel opening in the sinus was the residual non-dilated distal portion of vein. The other connection was the proximal part of vein draining the cerebellar hemisphere. Similar parallel venous channel as the recipient pouch in the transverse and sigmoid sinus dural fistulae has been described by Caragine et al [7].

Over the years, with recruitment of more feeders and increase in the calibre of the vessels, the flow into the varix might gradually increase. This resulted in outflow resistance due to thickening of the walls of the sinuses [3]. And the flow into the cerebellar cortical vein gradually reversed. This reflux resulted in arterialisation of the vein and formation of multiple varices in the cerebellar parenchyma. This was not associated with any sinus thrombosis or any symptoms of parenchymal venous congestion. However, the flow of blood was more towards the cortical vein than the sinus as could be interpreted from the preferential movement of the catheter tip during the procedure. A perfect division of the out flowing blood through both the routes might prevent a catastrophe for such a long duration.

The suddenness of the symptomatology can be explained by growing inflow of arterial blood by recruitment of fresh feeders. The walls of the veins and varices were strong enough to bear the pressure of arterial blood only up to a certain extent. In many cases reported in the literature the draining sinuses are blocked early in the course of disease leading to symptomatic cortical venous reflux [6]. However, in this case the sinuses remained patent till the end.

Partial TAE was performed in the acute phase for 


\section{DAVF at Confluence of Sinuses}

flow reduction as a palliative procedure. Though the patient was symptomatically better, it was thought that complete obliteration of the fistula was essential to prevent its growth and another hemorrhagic event. On repeated angiogram at our center, there was an increase in the shunt flow as compared to the postprocedural angiogram after first embolization. This indicates the malignant nature of the lesion and its growth potential even in 6 weeks. Thus, a need for radical treatment is strongly emphasized [8].

TVE for tentorial DAVF is to occlude the shunt points directly i.e., target embolization and thus restore the normal drainage pattern. Many authors believe in obliterating the patent sinus to achieve a radical cure, although this has been condemned by others $[9,10]$. TVE is essentially indicated when DAVF is supplied from arteries supplying cranial nerves, predominant ICA feeders and potential ECA-ICA collateral anatomises. Many authors do not advocate TVE due to complex venous anatomy in these cases, risk of venous perforation and incidence of venous hemorrhagic infarction. However, a meticulous and direct attack at the shunt point is the key to success in this procedure.

Early embolization of draining vein may lead to rupture of the varix directly associated with the fistula. But in the present case, we first embolized the draining vein. Because we were afraid that partial embolization of DAVF resulted in the residual reflux, as that vein was the source of SAH. Partial reduction of arterial flow into the fistula has decreased the risk of rupture of the varix during the embolization of the vein. After achieving complete occlusion of the cerebellar vein, we quickly embolized the varix with the coils. And residual feeders from the ECA could be embolized with the PVA particles through the transarterial route. Thus, a combination of various methods and a sandwich technique could help us attained complete obliteration.

Various evidences of congenital origin of the DAVF challenge its acquired theory of pathogenesis [3]. Isolated SAH is an uncommon presentation of DAVF and should not be missed. The DAVFs near the confluence are notorious and difficult to treat because of complex anatomy. They may fall into the combined category of tentorial medial DAVF and dural transverse sinus fistula according to the existing nomenclature.

Various methods of treatment for DAVF are described in the literature. Surgical, radiosurgical or endovascular approach might be one of the therapeutic options $[11,12]$. And endovascular approach might be the first treatment of choice. A combination of transar- terial and transvenous route might be essential for radical cure. Staged treatment to reduce the complication rates and to have a better understanding of the flow dynamics is recommended. Actually, DAVF might be cured by use of a combination of multiple methods. Endovascular embolization could be supplemented with surgical obliteration of residual lesion or radiosurgery in refractory cases $[13,14]$.

\section{Acknowledgement}

Rahul Gupta was actively corresponding to periprocedual management of this case, as Sugita Scholarship in Nagoya University supported by Department of Neurosurgery, Nagoya University Graduate School of Medicine. There was no conflict of interest.

\section{References}

1. Lasjaunias P, Chiu M, Brugge KT, Tolia A, Hurth M, Bernstein M. Neurological manifestations of intracranial dural arteriovenous malformations. J Neurosurg 1986;64:724-730

2. Picard L, Bracard S, Islak C, Roy D, Moreno A, Marchal JC, et al. Dural fistulae of the tentorium cerebelli: radioanatomical, clinical and therapeutic considerations. J Neuroradiol 1990;17:161-181

3. Miyachi S, Izumi T, Matsubara N, Naito T, Haraguchi K, Wakabayashi T. Mechanism of the formation of dural arteriovenous fistula: the role of the emissary vein. Interv Neuroradiol 2011;17:195-202

4. Chan ST, Weeks RD. Dural arteriovenous malformation presenting as cardiac failure in a neonate. Acta Neurochir (Wien) 1988;91:134-138

5. Pan HC, Sheehan J, Huang CF, Yang DY. Two consecutive dural arteriovenous fistulae in a child: a case report of successful treatment with gamma knife radiosurgery. Child Childs Nerv Syst 2007;23:1185-1190

6. Brown RD Jr, Wiebers DO, Nichols DA. Intracranial dural arteriovenous fistulae: angiographic predictors of intracranial hemorrhage and clinical outcome in nonsurgical patients. $J$ Neurosurg 1994;81:531-538

7. Caragine LP, Halbach VV, Dowd CF, Ng PP, Higashida RT. Parallel venous channel as the recipient pouch in transverse/ sigmoid sinus dural fistulae. Neurosurgery 2003;53:1261-1266

8. Pierot L, Chiras J, Meder JF, Rose M, Rvierez M, Marsault C. Dural arteriovenous fistulas of the posterior fossa draining into subarachnoid veins. AJNR Am J Neuroradiol 1992;13:315-323

9. Guedin P, Gaillard S, Boulin A, Condette-Auliac S, Bourdain F, Guieu S, et al. Therapeutic management of intracranial dural arteriovenous shunts with leptomeningeal venous drainage: report of 53 consecutive patients with emphasis on transarterial embolization with acrylic glue. J Neurosurg 2010;112:603-610

10. Shownkeen H, Yoo K, Leonetti J, Origitano TC. Endovascular treatment of transverse-sigmoid sinus dural arteriovenous malformations presenting as pulsatile tinnitus. Skull Base 2001;11:13-23

11. Kakarla UK, Deshmukh VR, Zabramski JM, Albuquerque FC, McDougall CG, Spetzler RF. Surgical treatment of high-risk intracranial dural arteriovenous fistulae: clinical outcomes and avoidance of complications. Neurosurgery 2007;61:447-457 


\section{Rahul Gupta, et al.}

12. Gross BA, Ropper AE, Popp AJ, Du R. Stereotactic radiosurgery for cerebral dural arteriovenous fistulas. Neurosurg Focus 2012;32:E18

13. Spiotta AM, Sivapatham T, Hussain MS, Hui FK, Moskowitz SI, Gupta R. Combined surgical and endovascular approach to a complex dural arteriovenous fistula involving the superior sagittal sinus and torcula. J Stroke Cerebrovasc Dis 2012;21:283-288

14. Natarajan SK, Ghodke B, Kim LJ, Hallam DK, Britz GW, Sekhar LN. Multimodality treatment of intracranial dural arteriovenous fistulas in the Onyx era: a single center experience. World Neurosurg 2010;73:365-379 